Plant Tissue Cult. \& Biotech. 23(2): 231-239, 2013 (December)

$\overline{\text { PTC\&B }}$

\title{
Development of Efficient Micropropagation Protocols of Elite Accessions of Jatropha curcas L.
}

\section{B. Naresh, G. Dhanasri, M Srikanth Reddy and Prathibha Devi Cherku*}

Plant Biotechnology and Molecular Genetics Laboratory, Department of Botany, Osmania University, Hyderabad 500007, India.

Key words: Jatropha curcas, Cotyledonary node, Axillary node, Shoot-tip, Adventitious shoots, Somatic embryos

\begin{abstract}
Efficient micropropagation protocols were developed from explants of two accessions of Jatropha curcas L. viz. Rain Shadow Area Development (RSAD) department and Karimnagar (KM). Adventitious shoots were regenerated with higher frequency on medium-A from cotyledonary node explants, than those from axillary node and shoot-tip explants. Shoots were also regenerated from calluses developed from cotyledonary node and shoot-tip explants on mediumB. Regenerated shoots were rooted on medium-G, transplanted to pots and later transferred to field. Somatic embryos developed on embryogenic calluses produced from cotyledonary node explants on medium- $C$ were converted to plantlets on medium-F and transferred to pots. Out of the 199 plants established in the Botanical Garden, 118 plants were developed from cotyledonary node explants, which indicates that they are the most suitable explants for micropropagation of Jatropha.
\end{abstract}

\section{Introduction}

Jatropha curcas L., a shrub or small tree (Euphorbiaceae) known as purging nut or physic nut has of late gained importance as a biodiesel yielding plant (Devi 2012).

The plant produces seeds from its third year and the decorticated seeds (kernels) contain $35-55 \%(\mathrm{w} / \mathrm{w})$ oil which is non-edible due to the presence of antinutritional factors like phorbol esters. Besides being used as biodiesel, the seed-oil has insecticidal, molluscicidal and fungicidal properties and the latex has medicinal value (Devi 2012).

"Author for correspondence: <prathi56@yahoo.com>. 
To meet the demand for supply of quality planting material, to raise plantations, various methods of propagation are being developed. Micropropagation by in vitro culture technology can be applied for Jatropha (Devi 2012). The present report contributes significantly to the development of a micropropagation protocol.

\section{Materials and Methods}

Seeds of two Indian accessions of Jatropha curcas viz. "RSAD" accession was obtained from Rain Shadow Area Development Department of Andhra Pradesh (A.P.) State Government, (Hyderabad, India), whereas the "KM" accession was collected from Venkatapur Village, Sircilla Mandal of Karimnagar District, A.P. at $18.43^{\circ} \mathrm{N} 79.15^{\circ} \mathrm{E}$ latitude. Both accessions were raised in the Experimental Botanical Garden, Department of Botany, Osmania University, Hyderabad, India.

Preliminary experiments were carried out with to selecting the most responsive explants. The hypocotyl and epicotyl explants were obtained from six-days-old seedlings, cotyledonary node $(\mathrm{CN})$ explants were obtained from 21days-old seedlings and the axillary node (AN), shoot-tip (ST), petiole (P) and leaf (L) explants from two-year-old mother plants growing in the Botanical Garden. The present study was carried out only with $\mathrm{CN}$, AN and ST explants since the other explants did not respond. The explants were thoroughly surface-sterilized using $10 \%(\mathrm{v} / \mathrm{v})$ hydrogen peroxide, $50 \%(\mathrm{v} / \mathrm{v})$ ethanol, $0.1 \%(\mathrm{w} / \mathrm{v})$ mercuric chloride and Tween-20, rinsed with sterile distilled water and inoculated with a pair of sterile forceps into $25 \times 150 \mathrm{~mm}$ culture tubes on autoclaved and cooled MS supplemented with $3 \%(\mathrm{w} / \mathrm{v})$ sucrose and solidified with $0.8 \%(\mathrm{w} / \mathrm{v})$ Phytaagar (Himedia). The $\mathrm{pH}$ of the medium was adjusted to 5.8 before autoclaving.

Forty explants were inoculated for each experiment with three replicates amounting to a total of 120 explants per accession. All the cultures were maintained in a sterile growth room with $16 \mathrm{hrs}$ photoperiod at $25 \pm 2^{\circ} \mathrm{C}$ with light intensity of $60 \mu \mathrm{Em}^{2} / \mathrm{s}$ and $50 \%$ relative humidity. Different culture media based on MS with supplementation of growth regulators were used. Citric acid (CA) $520.5 \mu \mathrm{M}(\mathrm{w} / \mathrm{v})$ was supplemented to all the media to prevent browning of tissue due to exudation of latex from explants. Compositions of the media (Table 1) are given below.

Medium-A: MS supplemented with IAA $(2,8 \mu \mathrm{M}), \mathrm{Kn}(13.93 \mu \mathrm{M})$, AS $(27.14$ $\mu \mathrm{M})$, Glutamine $(\mathrm{Glu})(684.2 \mu \mathrm{M})$ and CA $(520.5 \mu \mathrm{M})$. Medium-B: MS supplemented with 2, 4-D $(9.04 \mu \mathrm{M}), \mathrm{Kn}(4.6 \mu \mathrm{M})$, Glu $(684.2 \mu \mathrm{M})$ and CA (520.5 $\mu \mathrm{M})$. Medium-C: MS supplemented with 2, 4-D $(1.80 \mu \mathrm{M})$, BA $(1.7 \mu \mathrm{M})$, Glu $(684.2 \mu \mathrm{M})$ and $\mathrm{CA}(520.5 \mu \mathrm{M})$. 
Shoots which proliferated from all explants on medium-A were sub-cultured on medium-D for shoot elongation: Medium-D: MS supplemented with $\mathrm{GA}_{3}(5.7$ $\mu \mathrm{M})$, BA $(4.4 \mu \mathrm{M})$, Glu $(684.2 \mu \mathrm{M})$ and CA $(520.5 \mu \mathrm{M})$.

Calluses which developed from explants on medium-B were sub-cultured on medium-E for shoot induction: Medium-E: MS supplemented with NAA (2.68 $\mu \mathrm{M}), \mathrm{BA}(4.4 \mu \mathrm{M})$, Glu $(684.2 \mu \mathrm{M})$ and CA $(520.5 \mu \mathrm{M})$.

Explants with somatic embryos (from medium-C) were transferred to medium-F for conversion of somatic embryos to plantlets.

Medium-F: MS supplemented with BA $(6.6 \mu \mathrm{M})$, Glu $(684.2 \mu \mathrm{M})$ and CA $(520.5 \mu \mathrm{M})$.

Healthy, well developed and elongated shoots $(3 \mathrm{~cm})$ were transferred to medium-G for root induction: Medium-G: Half strength MS supplemented with IBA $(14.7 \mu \mathrm{M})$, Glu $(684.2 \mu \mathrm{M})$ and CA $(520.5 \mu \mathrm{M})$.

Healthy plantlets with well-developed roots were transferred to pots and gradually acclimated in the glasshouse in the shade for two weeks by enclosing the pots in polythene bags. Subsequently, the plants were transferred to the sunny areas of the glasshouse and later to the field.

Each experiment was repeated twice with three replicates/treatments Data pertaining to the study was recorded, statistically analyzed and presented as mean \pm standard error of the three replicates.

Histological studies were conducted to identify the morphogenetic pathway (organogenesis or somatic embryogenesis) either directly from explants or indirectly from callus according to Luna (1998). The materials were fixed in alcohol and glacial acetic acid $3: 1$. The fixed tissue was passed through a series of dehydration steps in tertiary butyl alcohol (30-70\%), and xylene was used as a clearing agent to make the tissue translucent. The material was then impregnated with paraffin $\left(56-58^{\circ} \mathrm{C}\right)$ and placed in the embedding unit (Electron Corporation, model Shandon Histocenter-3). The tissue was processed by Citadel-2000 Thermo Shandon and stained with haemotoxylin and eosin, using an automated Leica Auto stainer-Xl. These blocks were mounted and trimmed using a microtome (Leica model RM 2155) for very thin microsections of $6-8 \mu \mathrm{m}$. The sections were mounted on slides, dewaxed and observed under the microscope- (Leitz Diaplan) and microphotographs of different stages of shoot-bud induction or somatic embryogenesis were obtained with a digital camera. 


\section{Results and Discussion}

The present study reports rapid, reliable and reproducible protocols for efficient micropropagation of two accessions of J. curcas. Although plant regeneration was reported previously (Sujatha and Mukta 1996, Jyothi et al. 2000, Wei et al. 2004, Rajore and Batra 2005, Sujatha et al. 2005, Nannapat et al. 2006, Datta et al. 2007), an attempt was made to achieve a higher frequency of regeneration to enhance micropropagation. As selection of explants is an important criterion, different explants were used presently and encouraging results obtained with $\mathrm{CN}, \mathrm{AN}$ and ST explants through direct regeneration, and through callus and via somatic embryos.

Adventitious shoot-buds developed from all three explants of RSAD and KM accessions by the fourth week on medium-A (Fig. 1a, b). The most efficient shootbud induction was recorded from $\mathrm{CN}$ explants viz. $95 \pm 0.3$ and $86 \pm 0.6$ in "RSAD" and "KM" accessions respectively followed by AN explants viz. $81 \pm 0.3$ and $76 \pm 0.2$ in "RSAD" and "KM" accessions, respectively and ST explants viz. $60 \pm 0.4$ and $64 \pm 0.8$ in "RSAD" and "KM" accessions, respectively (Table 2). The cultured shoots proliferated to about $5 \mathrm{~cm}$ in length with fully expanded leaves by the end of the fourth week after transfer to medium-D. Shoots could be regenerated with a higher efficiency from CN explants $(94 \pm 0.3$ in "RSAD" and $92 \pm 0.3$ in "KM" accessions than those from AN and ST explants and were also induced to develop roots on medium-G with a higher efficiency. Although direct shoot regeneration was earlier reported from AN explants (Sujatha et al. 2005, Nannapat et al. 2006, Datta et al. 2007), ST explants (Rajore and Batra 2005) and leaf segments (Sujatha et al. 2005), the present efficiency achieved is considerably higher.

Green nodular callus developed from $\mathrm{CN}$ and ST explants on medium-B (Fig. 1d) with callusing frequency of $38 \pm 0.5$ to $69 \pm 0.4$ (Table 2). Shoot-buds regenerated from these calluses (confirmed by histological studies) by the end of the second week on medium-E proliferated into $3-4 \mathrm{~cm}$ long shoots with fully expanded leaves by the end of the fourth week and developed roots on medium$\mathrm{G}$ (Fig. 1d, e). A higher frequency of shoot regeneration was recorded from $\mathrm{CN}$ explants ( $30 \pm 0.3$ and $38 \pm 0.4$ in "RSAD" and "KM" accessions respectively), than those of ST explants ( $24 \pm 0.3$ and $28 \pm 0.5$ in "RSAD" and "KM" accessions, respectively) (Table 2). There are no reports of shoot regeneration via callus from the CN or ST explants. However, it was reported from leaf, hypocotyl, and petiole explants (Sujatha and Mukta 1996) and epicotyl explants (Wei et al. 2004).

Green and globular proembryos developed from embryogenic callus from $\mathrm{CN}$ explants on medium- $\mathrm{C}$ further differentiated into heart-shaped somatic embryos by the end of the third week (Fig. 1e, f). After the conversion of these 
Development of Efficient Micropropagation Protocols

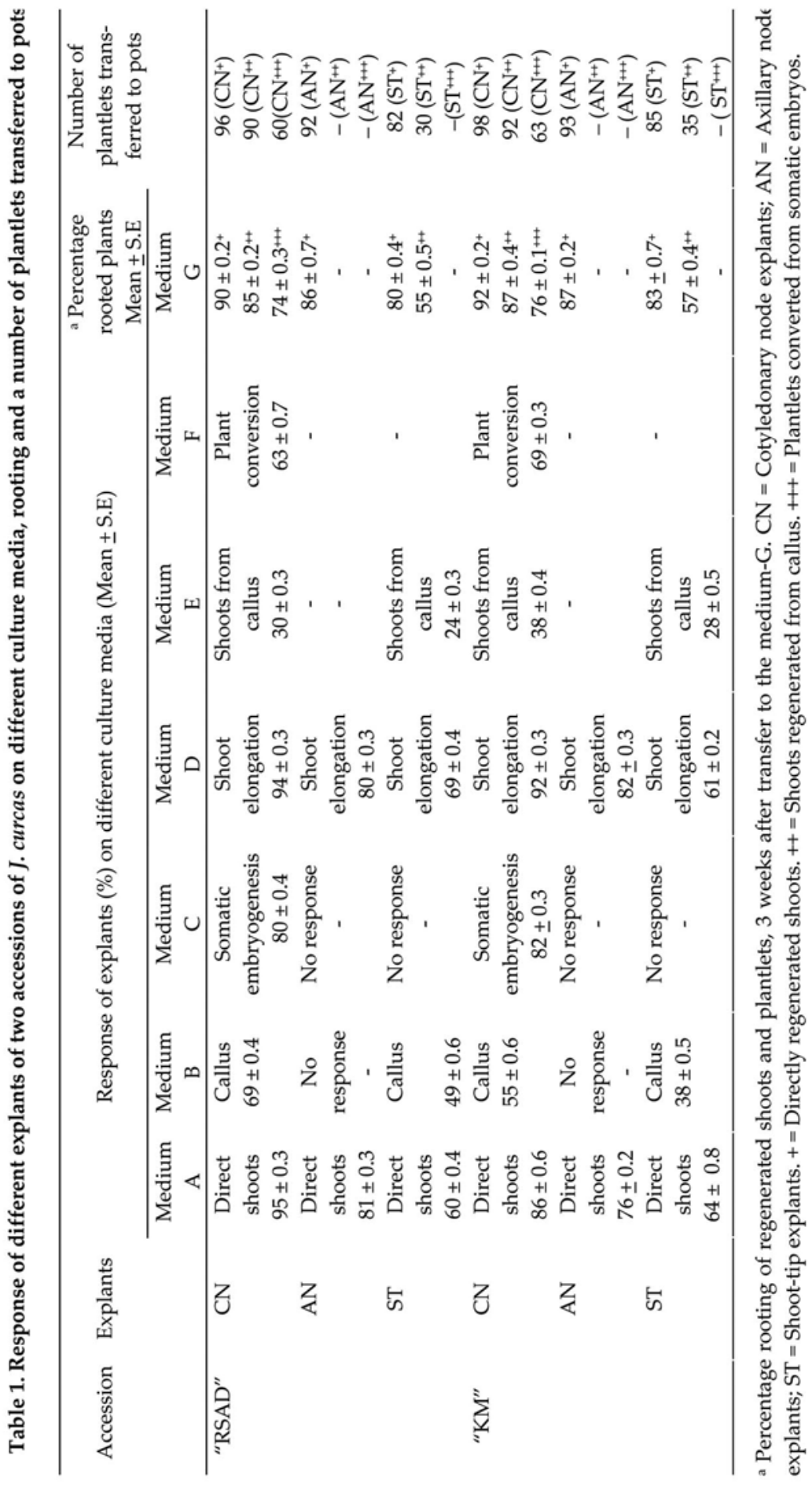


embryos into plantlets on medium-F (Fig.1g), they were transferred to medium$\mathrm{G}$ where they developed stronger roots in eight weeks. The frequency of conversion of somatic embryos into plantlets was higher in "KM" accession (69 \pm 0.3 ) than that of "RSAD" accession (63 \pm 0.7$)$ (Table 2). Plantlet regeneration from leaf explants via somatic embryogenesis was reported by Jyothi et al. (2000). However, the $\mathrm{H}, \mathrm{E}, \mathrm{P}$ and L explants did not respond to in vitro culture presently. This might have occurred due to innate differences in the concentration of endogenous growth regulators based on varietal differences and age of the seedlings.

Table 2. Survival of in vitro developed plantlets of two accessions of $J$. curcas in the Botanical Garden of Osmania University.

\begin{tabular}{|c|c|c|c|c|c|}
\hline Accession & Explant & $\begin{array}{l}\text { a Percentage } \\
\text { survival of } \\
\text { plants in G } \\
\text { Mean } \pm \text { S.E }\end{array}$ & $\begin{array}{c}\text { Number of } \\
\text { plantlets } \\
\text { transferred to } \\
\text { BG }\end{array}$ & $\begin{array}{c}\text { bercentage } \\
\text { survival } \\
\text { of plants in BG } \\
\text { Mean } \pm \text { S.E }\end{array}$ & $\begin{array}{l}\text { Number of } \\
\text { plants } \\
\text { established in } \\
\text { BG }\end{array}$ \\
\hline \multirow{9}{*}{ “RSAD” } & $\mathrm{CN}^{+}$ & $68 \pm 0.6$ & 65 & $55 \pm 0.6$ & 36 \\
\hline & $\mathrm{CN}^{++}$ & $40 \pm 0.2$ & 36 & $36 \pm 0.6$ & 13 \\
\hline & $\mathrm{CN}^{+++}$ & $35 \pm 0.8$ & 21 & $33 \pm 0.6$ & 7 \\
\hline & $\mathbf{A N}^{+}$ & $44 \pm 0.7$ & 40 & $53 \pm 0.7$ & 21 \\
\hline & $\mathbf{A N}^{++}$ & - & - & - & - \\
\hline & $\mathrm{AN}^{+++}$ & - & - & - & - \\
\hline & $\mathrm{ST}^{+}$ & $37 \pm 0.4$ & 30 & $50 \pm 0.2$ & 15 \\
\hline & $\mathrm{ST}^{++}$ & $27 \pm 0.6$ & 8 & $25 \pm 0.6$ & 2 \\
\hline & $\mathrm{ST}^{+++}$ & - & - & - & - \\
\hline \multirow{9}{*}{ “KM" } & $\mathrm{CN}^{+}$ & $70 \pm 0.5$ & 69 & $57 \pm 0.3$ & 39 \\
\hline & $\mathrm{CN}^{++}$ & $42 \pm 0.6$ & 39 & $38 \pm 0.4$ & 15 \\
\hline & $\mathrm{CN}^{+++}$ & $37 \pm 0.6$ & 23 & $35 \pm 0.7$ & 8 \\
\hline & $\mathbf{A N}^{+}$ & $45 \pm 0.8$ & 42 & $55 \pm 0.5$ & 23 \\
\hline & $\mathbf{A N}^{++}$ & - & - & - & - \\
\hline & $\mathrm{AN}^{+++}$ & - & - & - & - \\
\hline & $\mathrm{ST}^{+}$ & $39 \pm 0.5$ & 33 & $51 \pm 0.8$ & 17 \\
\hline & $\mathrm{ST}^{++}$ & $29 \pm 0.6$ & 10 & $33 \pm 0.3$ & 3 \\
\hline & $\mathrm{ST}^{+++}$ & - & - & - & - \\
\hline
\end{tabular}

${ }^{a}$ Percentage survival of the transplanted plantlets 3 weeks after transfer to G. bPercentage survival of the transplanted plantlets two weeks after transfer to BG. $\mathrm{CN}=$ Cotyledonary node explants; $\mathrm{AN}=$ Axillary node explants; ST $=$ Shoot-tip explants. $+=$ Directly regenerated shoots. $++=$ Shoots regenerated from callus. $+++=$ Plantlets converted from somatic embryos. $G=$ Glasshouse; $B G=$ Botanical garden.

All the regenerated shoots could be induced to develop roots by the end of third week with the highest efficiency recorded for directly regenerated shoots of all the three explants. Percentage root induction recorded from shoots developed from CN explants ranged from $74 \pm 0.3$ to $92 \pm 0.2$ (Table 2). The percentage root induction of shoots obtained directly from AN explants was $86 \pm 0.7$ and $87 \pm 0.2$, respectively. Whereas, percentage root induction of shoots developed from ST 
explants both directly and through callus ranged from $55 \pm 0.5$ to $83 \pm 0.7$, a higher efficiency of rooting was recorded from directly regenerated shoots (Table 2).

Plantlets with healthy shoots and well-developed roots were transferred to pots and could be acclimated in the glasshouse by three weeks (Fig. 1h). Survival was more in case of directly regenerated plantlets than those regenerated via callus or somatic embryos (for all the explants) in both accessions. Further, those regenerated from $\mathrm{CN}$ explants had the highest survival rate compared to those regenerated from ST and AN explants in both the accessions (Table 3). The most efficient explant was the $\mathrm{CN}$ and the most efficient route of plant regeneration was direct shoot regeneration. The protocols were largely accession independent.
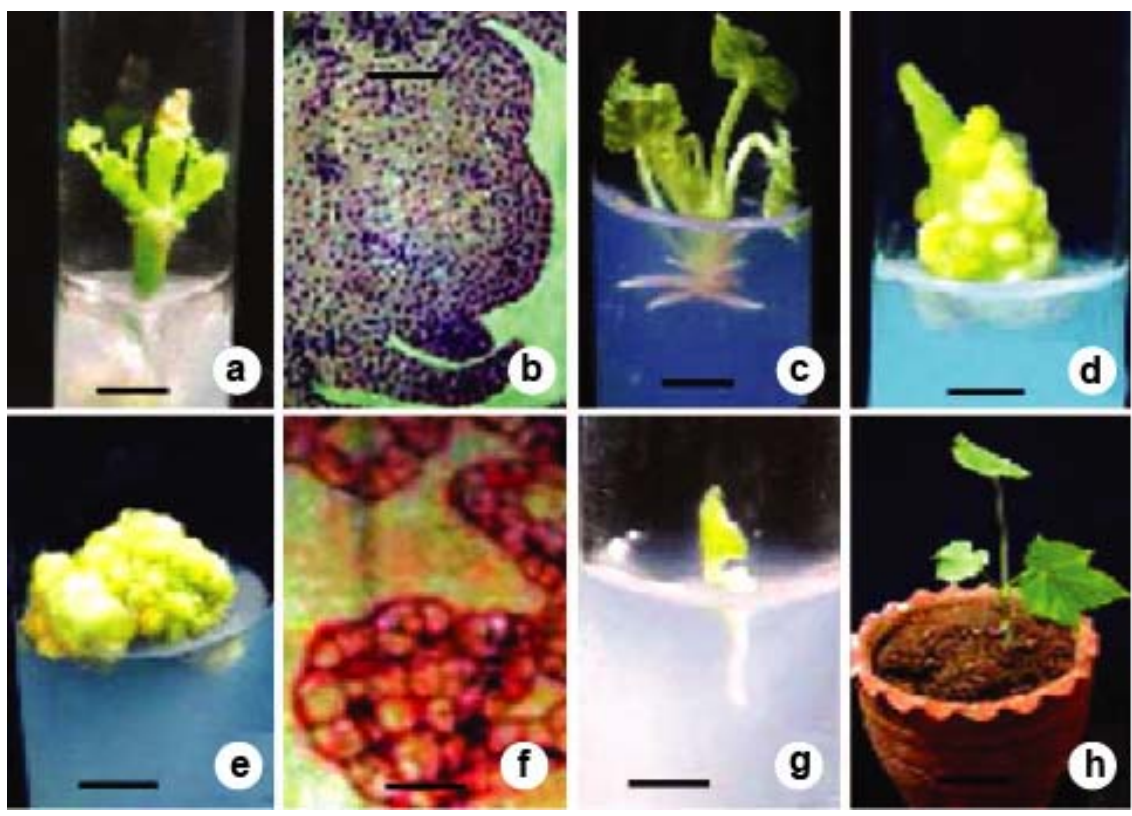

Fig. 1a-h. Plant regeneration response of CN, AN and ST explants of two accessions of Jatropha curcas cultured on various culture media in vitro. (a) Proliferation of multiple shoot buds from AN explants of "RSAD" genotype by the end of the second week after transfer to medium-D (Bar: $1 \mathrm{~cm})$. (b) A histological section of the callus tissue with shoot-organogenesis for the development of shoot-buds (Bar: $100 \mu \mathrm{m}$ ). (c) Four-week-old shoots with leaves (produced from CN explant of "RSAD" accession by direct shoot regeneration), rooted on medium-G (Bar: $1 \mathrm{~cm})$. (d) Green nodular calli developed from ST explant of "KM" accession on medium-B (Bar: $1 \mathrm{~cm}$ ). (e) Proembryos from the embryogenic callus that was produced from CN explants of "KM" accession on medium-C (Bar: $1 \mathrm{~cm})$. (f) A histological section of a heart-shaped somatic embryo (indicated by arrow) developed from $\mathrm{CN}$ explant of "KM" accession, after the third week of culture (Bar: $100 \mu \mathrm{m}$ ). (g) Plantlets of "RSAD" accession converted from somatic embryos on medium-G (Bar: $1 \mathrm{~cm}$ ). (h) A plantlet of "KM" accession transferred to a pot and gradually acclimated in the glasshouse before its transfer to the Botanical Garden (Bar: $3.2 \mathrm{~cm}$ ). 
Out of a total of 200 plantlets of "RSAD" accession and 216 plantlets of "KM" accession transferred to the Botanical Garden, only 199 plantlets (94 of "RSAD" and 105 of "KM" accessions) were successfully established over a period of 12 weeks (Table 2). Plantlets could be directly regenerated in 14 weeks, via callus in 17 week and via somatic embryos in 19 week.

Efficient plantlet regeneration from $\mathrm{CN}$ is a first report in Jatropha. The use of the amenable $\mathrm{CN}$ explants paves the way for successful micropropagation of Jatropha. Cotyledons are good explants for shoot regeneration since the meristem cells are easily formed at their base and they can be directed towards development of shoot primordia with the correct hormonal balance (Wei et al. 2004). Further, the percentage of plantlet regeneration (up to rooting) achieved presently from AN and ST explants (86-87\%) is also suited for micropropagation. Differential response of the explants to the shoot induction medium may be attributed to the correlative interaction between the type of tissue and the concentration of endogenous growth regulators (George 1993).

Shoot regeneration via callus (from $\mathrm{CN}$ and ST explants) and plantlet regeneration via somatic embryogenesis (from $\mathrm{CN}$ explants) are also being reported for the first time in J. curcas although somatic embryogenesis was reported from leaf explants (Jyothi et al. 2000, Jha et al. 2007). The use of ST and CN explants was prompted by our earlier successful studies (Devi et al. 2000, Kumar et al. 2004). Growth regulators are important factors, which can selectively influence the genes to trigger the differentiation of cells in culture (Thorpe 1983). Although there are some earlier reports of use of other combinations (Sujatha and Mukta 1996, Jyothi et al. 2000, Wei et al. 2004, Rajore and Batra 2005, Sujatha et al. 2005, Nannapat et al. 2006, Datta et al. 2007), the present study reveals that the combination of IAA with Kn and AS elicited a very high response of 7-9 shoot-buds from CN, AN and ST explants. The supplementation of AS resulted in a synergistic effect with $\mathrm{Kn}$ in accordance with the report of Rajore and Batra (2005).

Although, rooting has been efficiently induced presently on half strength MS with IBA $(14.7 \mu \mathrm{M})$, Glu $(684.2 \mu \mathrm{M})$ and CA $(520.5 \mu \mathrm{M})$ (medium-G), it needs to be further improved to increase the survival percentage of in vitro regenerated Jatropha plants on the field. The protocols developed presently could be used for large scale micropropagation for development of planting material of Jatropha.

\section{Acknowledgements}

Authors sincerely acknowledge the University Grants Commission (UGC), India for the financial assistance granted for a major research project to Prof. Prathibha Devi Cherku. 


\section{References}

Datta MM, Mukherjee P, Ghosh B and Jha TB (2007) In vitro clonal propagation of biodiesel plant (Jatropha curcas L.). Curr. Sci. 93: 1438-1442.

Devi P (2012) Status of Bioenergy Research and Jatropha in India: A Review. In: Carels N., Sujatha M., Bahadur B. (Eds.) Jatropha, Challenges for a New Energy Crop, Vol. I, Springer Science+Business Media New York 1: 31-51.

Devi P, Zhong $\mathbf{H}$ and Sticklen MB (2000) In vitro morphogenesis of pearl millet [Pennisetum glaucum (L.) R.Br.] and efficient production of multiple shoots and inflorescence from shoot apices. Plant Cell Rep. 19: 546-550.

George EF (1993) Plant propagation by tissue culture Part1. The Technology. Exegetics

Limited. Edington, Wilts UK. pp. 574

Jha TB, Mukherjee P and Datta MM (2007) Somatic embryogenesis in Jatropha curcas L., an important biofuel plant. Plant Biotechnol. Rep. 1: 135-140.

Jyothi S, Batra A and Ali DJ (2000) An expeditious method for regeneration of

somatic embryos in Jatropha curcas L. Phytomorphol. 50 (384): 239-242.

Kumar SM, Kumar BK, Sharma KK and Devi P (2004) Genetic transformation of pigeonpea with rice chitinase gene. Plant Breed. 123: 485-489.

Luna LG (1998) Manual of histological staining methods of AFLP IIIEd, Mc Graw Hill.

Nannapat T, Chockpisit T and Aree T (2006) In vitro induction of shoots and roots from Jatropha curcas L. explants. J Horti Sci \& Biotech. 83: 106-112.

Rajore S and Batra A (2005) Efficient Plant regeneration via shoot tip explants in Jatropha curcas L. J Plant Biochem Biotech. 14: 73-75.

Sujatha M and Mukta N (1996) Morphogenesis and plant regeneration from tissue cultures of Jatropha curcas. Plant Cell Tiss. Org. Cult. 44: 135-141.

Sujatha M, Makkar HPS and Becker K (2005) Shoot bud proliferation from axillary nodes and leaf sections of non-toxic Jatropha curcas L. Plant Gr. Reg. 47:83-90

Thorpe TA (1983) Morphogenesis and regeneration in tissue culture. Beltsville Sym. Agric. Res. 7: 285-303.

Wei Q, Lu W, Da LY, Pan SL Xu Y, Tang L and Chen F (2004) Plant regeneration from epicotyl explant of Jatropha curcas. J. Plant Physiol. Mol. Biol. 30 (4): 475-478. 